> Les carcinomes sont composés, d'une part, de cellules épithéliales malignes et, d'autre part, du microenvironnement tumoral, encore appelé stroma tumoral. Cette revue, non exhaustive, se focalisera sur les évènements cellulaires et moléculaires clés impliquant les cancerassociated fibroblasts (CAF) et la matrice extracellulaire (MEC) dans le remodelage du stroma au cours de l'invasion tumorale. Nous aborderons également la place du stroma dans la cancérologie d'aujourd'hui, les avancées récentes qui ont été faites et les retombées thérapeutiques potentielles qui en découlent. <

Les protagonistes du dialogue entre les cellules épithéliales et le stroma

Les organes adultes sont composés de deux compartiments, I'un épithélial et l'autre conjonctif, encore appelé stroma. Ces deux compartiments établissent des interactions/dialogues réciproques strictement régulés par des signaux de différentes natures (mécaniques, moléculaires), de façon à maintenir l'architecture et la taille de l'organe, et à en assurer la fonction propre [1-3]. Ces deux compartiments sont délimités par une sorte de barrière appelée membrane ou lame basale. Ainsi, dans des conditions normales, les cellules épithéliales ne sont tolérées dans les tissus conjonctifs périphériques que de façon transitoire, au cours de processus biologiques assurant la morphogenèse/ croissance tissulaire (par exemple pendant le développement postnatal de la glande mammaire), ou dans des processus de réparation/reconstruction tissulaire (blessures, involution de l'utérus après la parturition et du sein après la lactation). Au terme de ces processus, les cellules épithéliales résiduelles qui persistent de façon aberrante dans le compartiment conjonctif sont alors éliminées par une apoptose de type anoïkis, assurant ainsi l'homéostasie tissulaire. II existe donc

Cet article fait partie du numéro thématique publié par médecine/sciences en avril 2014 et intitulé « Microenvironnement tumoral ».

\section{Le stroma tumoral}

\section{Un terreau fertile pour la cellule cancéreuse}

\section{Émilie Buache, Marie-Christine Rio}



Institut de génétique et de biologie moléculaire et cellulaire (IGBMC), département de génomique fonctionnelle et cancer, 1, rue Laurent Fries, BP 10142, 67404 IIIkirch Cedex, France; CNRS UMR 7104, Inserm U964, université de Strasbourg, 67404 III kirch Cedex, France. rio@igbmc.fr

un mécanisme qui fait intervenir des signaux forts en provenance des tissus environnants, et limite, voire empêche, la survie/implantation de cellules épithéliales. Dans un contexte normal, le stroma régule négativement le phénotype malin, ce qui explique l'existence relativement fréquente de tumeurs dormantes (occultes) [4]. On peut donc dire qu'il existe une sorte de «dominance » du compartiment stromal sur le compartiment épithélial.

Dans ce contexte, le développement d'un carcinome invasif, c'est-àdire de cellules épithéliales malignes, dans les tissus environnants, n'aura lieu que si les signaux d'origine stromale sont modifiés. Les cellules cancéreuses invasives vont œuvrer dans ce sens. Non seulement elles s'adaptent elles-mêmes pour circonvenir les signaux de mort venant des tissus périphériques normaux, mais elles stimulent aussi les cellules normales résidentes de ces tissus, et induisent des signaux permettant leur survie dans ce milieu hostile. Ceci se traduit par un remodelage tissulaire, encore appelé desmoplasie, qui commençe dès les premières étapes d'invasion locale et conduit à une déstructuration sévère du tissu. II y a altération/activation de certaines cellules et de la matrice extracellulaire (MEC), et formation d'un stroma constitué, favorable à la progression tumorale puis à la colonisation de l'individu dans sa globalité. Ainsi, la cellule cancéreuse, bien qu'issue du même individu, va se comporter comme un parasite, en ce sens qu'elle va vivre aux dépens du stroma qui représente l'«hôte » $[31,32](\rightarrow)$

Dans cette revue, nous nous focaliserons, d'une part, sur le stroma tumoral présent au niveau des tumeurs solides primaires et, d'autre part, sur les fibroblastes responsables en grande partie de la formation de ce stroma, et, enfin, sur leurs fonctions respectives [33] $(\rightarrow)$.

$(\rightarrow)$ Voir les Synthèses de W.H. Fridman et C. Sautès-Fridman, et de V. Provot, pages 359 et 366 de ce numéro

$(\rightarrow)$ Voir la Synthèse de J. Albrengues et al., page 391 de ce numéro 


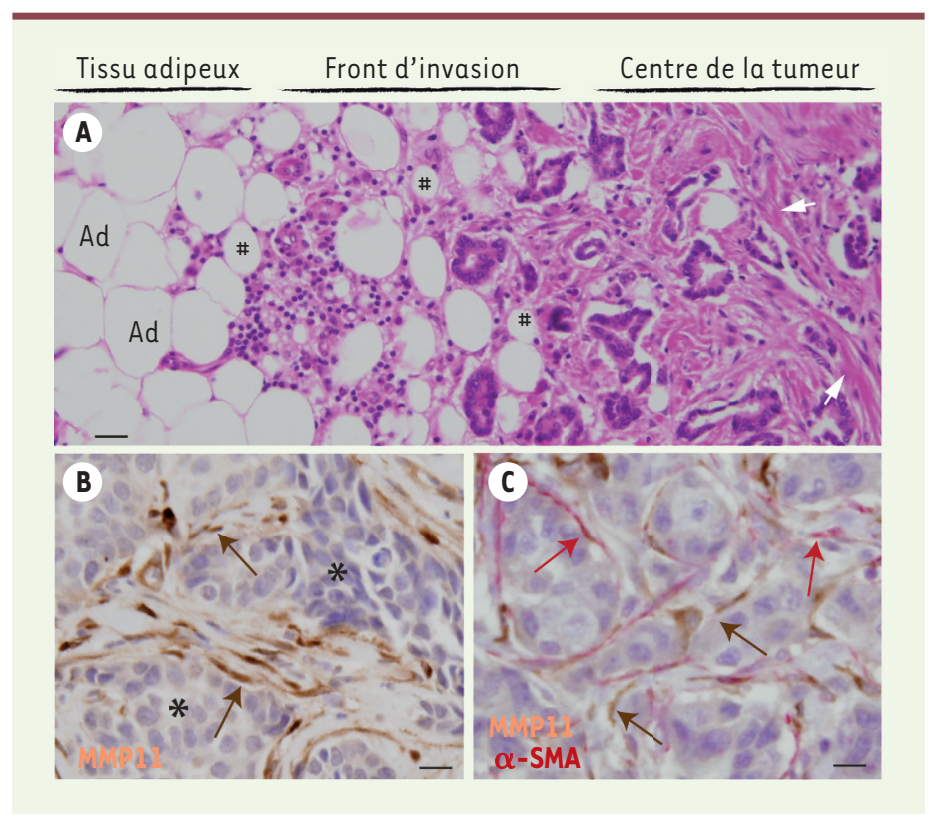

Figure 1. Changement de l'organisation tissulaire globale en fonction du stade de la tumeur. A. Histologie d'une coupe de cancer du sein illustrant le remodelage tissulaire au cours de la progression tumorale. L'image peut être sous-divisée arbitrairement en trois parties : le tissu adipeux d'origine, le front d'invasion de la tumeur et le centre de la tumeur. II s'agit d'un processus dynamique commençant dès les premiers évènements invasifs au front d'invasion. La désorganisation s'accentue ensuite avec la progression tumorale, l'architecture tissulaire montrant de moins en moins de ressemblance avec celle du tissu de départ. D'un tissu dominé par les adipocytes ( $\mathrm{Ad}$ ), on passe par un front d'invasion contenant des cellules mésenchymateuses variées (dont des CAA [cancer associated adipocytes] $[\#]$ ) pour aboutir au centre de la tumeur à un stroma constitué où prédominent de larges travées de stroma (flèches blanches) constituées de MEC et de CAF. Barre d'échelle : $100 \mu \mathrm{m}$. B. Marquage immunohistochimique de CAF présents au centre de la tumeur à l'aide d'un anticorps dirigé contre la MMP1l. La MMP1l est surexprimée par des CAF (flèches brunes) présents en périphérie d'îlots de cellules cancéreuses (astérisques). Les noyaux des cellules sont colorés en bleu. Barre d'échelle : $50 \mu \mathrm{m}$. C. Co-marquage immunohistochimique permettant de visualiser simultanément deux types de CAF, I'un en rouge correspondant à des myofibroblastes exprimant l' $\alpha$-SMA (alpha smooth muscle actin ; flèches rouges) et l'autre marqué en brun exprimant la MMP1l (flèches brunes). Barre d'échelle : $50 \mu \mathrm{m}$.

\section{Constitution du stroma tumoral}

Le stroma ou microenvironnement tumoral est constitué de populations diverses de cellules normales d'origine mésenchymateuse, incluant des fibroblastes, des cellules endothéliales, des cellules inflammatoires (macrophages, neutrophiles, lymphocytes), des cellules souches mésenchymateuses et des préadipocytes/adipocytes qui sont inclus dans une MEC $[1-3,34](\rightarrow)$. Le stroma tumoral diffère du tissu conjonctif normal. En effet, d'une part, les compartiments épithélial et conjonctif ne sont plus délimités par une membrane basale intacte et, d'autre part, il possède une composition propre (Figure 1).
La nature du microenvironnement tumoral est dépendante de la composition d'origine de l'organe dans lequel se développe la tumeur (sein, côlon, prostate, etc.) et d'évènements dépendants de la tumeur conduisant à son altération. De plus, l'intensité des réactions stromales varie d'un organe à l'autre. Ces réactions stromales sont notamment très importantes dans les cancers touchant le sein, le pancréas et le côlon [32-34].

Enfin, le stroma tumoral est sujet à une certaine dynamique puisque, pour une même tumeur, il variera énormément au cours des différentes étapes du processus tumoral, conduisant ainsi à son adaptation au bon vouloir de la cellule cancéreuse : (1) adaptation dans le temps, entre les phases précoces d'invasion locale pendant lesquelles des cellules cancéreuses «pionnières » vont rencontrer les cellules normales résidentes de proximité et initier les premières réactions stromales, et les phases de croissance des tumeurs primaires, quand les phénomènes de desmoplasie sont déjà bien avancés; (2) adaptation dans l'espace, entre invasion locale et invasion à distance selon le lieu de développement des métastases (poumon, cerveau ou autres organes; niches prémétastatiques) [1-3].

\section{Activation de cellules normales de l'hôte par les cellules cancéreuses}

La desmoplasie se traduit tout d'abord par l'activation de cellules normales résidentes de l'organe touché. Plusieurs évènements s'avèrent décisifs pour l'avenir des cellules cancéreuses. Ainsi, un système vasculaire se développe de façon à subvenir aux besoins en nutriments des cellules cancéreuses et permettre la croissance tumorale au-delà d'une certaine taille. C'est ce que l'on appelle l'angiogenèse tumorale $[5,35,36](\rightarrow)$. En présence de tissus adipeux, un autre fait marquant est la quasi

$(\rightarrow)$ Voir la Synthèse de S. Azzi et J. Gavard, page 408 de ce numéro disparition des adipocytes au contact des cellules cancéreuses invasives au profit d'adipocytes de taille réduite, les CAA (cancer associated adipocytes) [37] $(\rightarrow)$ et de CAF (cancer-associated fibroblast)

\section{$(\rightarrow)$ Voir la Synthèse} de V. Laurent et al., page 398 de ce numéro (Figure 1A) [6-8].

Une fois le processus de desmoplasie bien engagé, les cellules stromales les plus fréquentes sont des cellules de type fibroblastique appelées CAF ou TAF (tumorassociated fibroblasts) (Figure 1). Les CAF présentent des caractéristiques différentes des fibroblastes normaux, notamment un profil d'expression génique qui leur est propre (Figure 2). Leur phénotype est dit 
activé [9]. Il a été proposé que ce dernier soit acquis en deux phases: la première, réversible, suivie d'une étape irréversible. Ainsi, il y a une sorte d'empreinte de la cellule cancéreuse sur les CAF. La présence de mutations somatiques dans les CAF est discutée $[10,11]$.

\section{Les CAF : d'où viennent-ils, que font-ils ?}

Il est actuellement bien admis que le stroma tumoral ne renferme pas que des myofibroblastes, mais une population hétérogène de $C A F$, ce qui pose la question de leur(s) origine(s) [33]. Bien que leur caractérisation précise reste à élucider, plusieurs données ont été acquises dans ce domaine. Ainsi, les CAF peuvent tout d'abord provenir de la multiplication de fibroblastes résidents. Plusieurs autres types cellulaires d'origine mésenchymateuse peuvent être mis à contribution, dont notamment les péricytes, les préadipocytes voire les adipocytes, les fibroblastes sénescents, des cellules souches, ou encore des cellules issues de la moelle osseuse $[8,11,12]$. Ces différentes provenances pourraient expliquer les variations phénotypiques du profil moléculaire des CAF. En effet, de nombreux facteurs sont exprimés différentiellement dans les CAF par rapport aux fibroblastes des tissus normaux, et jouent un rôle dans la progression tumorale (Figure 2) $[11,12]$.

D'un point de vue fonctionnel, les CAF influencent le devenir des cellules cancéreuses [1-3]. Ils peuvent émettre des signaux agissant de façon autocrine ou paracrine, voire endocrine. Ils synthétisent notamment de nombreuses cytokines/chémokines, des facteurs de croissance, des protéines du cytosquelette, des protéines de la MEC et des enzymes. L'impact du stroma sur la progression tumorale a été appréhendé à l'aide de modèles murins. La co-injection de fibroblastes et de cellules cancéreuses favorise la progression tumorale [9]. Ainsi, nous avons montré chez l'homme que l'expression ectopique de la métalloprotéase matricielle 11 (MMPll ou stromélysine-3) dans les CAF (Figures IB-C) était associée à un pronostic péjoratif [13]. Un argument de poids est aussi apporté par l'observation que les fibroblastes issus de souris déficientes en MMPll perdent leur capacité à promouvoir le développement de tumeurs [14].

\section{La matrice extracellulaire}

La MEC est la composante non cellulaire du stroma. Elle est constituée principalement d'un grand nombre de protéines de grandes tailles, telles que les collagènes, les laminines, les fibronectines et les protéoglycanes qui forment un réseau dense. Normalement, les fibroblastes sont les pourvoyeurs principaux de la MEC. De très bonnes revues détaillées répertoriant la nature des différents composants de la MEC existent déjà dans la littérature [15] ; cet aspect ne sera donc pas abordé ici. La mise en place de la MEC se fait par étapes. Ainsi, ses composants comme les collagènes sont synthétisés, sécrétés, déposés, assemblés et/ou remodelés en fonction des besoins. La MEC a une fonction de maintien de la structure tissulaire via des contraintes physiques, qui facilitent les communications entre cellules et servent de support pour l'attachement des cellules et leur migration. Dans un contexte tumoral, les CAF sont responsables de la surproduction de composants matriciels qui modifie son architecture et les contraintes mécaniques [16, 17]. Ainsi, le tissu à proximité de la tumeur est «rigide » comparé au tissu non tumoral ; cela est dû au dépôt de grandes quantités de collagène par les CAF [18]. Les modifications de forces/tensions entraînent des réactions mécaniques se traduisant ensuite en fonctions biologiques [33]. De plus, les CAF affectent le microenvironnement tumoral par la synthèse et la sécrétion de protéases, certaines spécialisées dans la dégradation de la MEC, dont notamment les MMP. Ainsi, la MEC est une structure non statique, dont le paysage et la fonction varient en regard des zones d'invasion.

La MEC présente de nombreuses autres fonctions. Elle sert de réservoir à un certain nombre de facteurs solubles, souvent sous forme de profacteurs (pro-TGF $\beta$ [pro-transforming growth factor $\beta$ ], IGFBP] [insulin-like growth factor binding protein 1]). De telles molécules, une fois activées, sont capables d'induire en aval une ou plusieurs voies de signalisation par le biais d'interactions avec d'autres protéines, le plus souvent des récepteurs membranaires. Cela conduit in fine à des modifications transcriptionnelles au sein des cellules cibles stromales et/ou tumorales. Les protéines nouvellement formées peuvent, à leur tour, envoyer des signaux vers ces mêmes cellules [1-3].

\section{Les métalloprotéases matricielles}

Autrefois, il était admis que les MMP étaient produites par les cellules cancéreuses elles-mêmes, qui, de cette façon, se frayaient un chemin dans le tissu environnant et les colonisaient. Cependant, à la fin des années 1980, des études réalisées sur des tumeurs humaines ont montré que certaines MMP étaient restreintes au stroma, comme par exemple la MMPll (Figures 1B-C) [13]. Un nouveau concept a alors émergé proposant que les MMP soient au contraire produites par les cellules normales périphériques de type fibroblastique [13]. II est maintenant bien établi que les fibroblastes - et les CAF en particulier - sont la source majeure de la plupart des MMP, renforçant la notion de collaboration/dialogue entre cellules cancéreuses et cellules stromales.

Ces enzymes ont une fonction majeure dans le remodelage de la MEC et sont capables de dégrader au moins un composant de la MEC. Elles sont inhibées par leurs inhibiteurs naturels, les TIMP (inhibiteurs tissulaires de MMP). De plus, ces clivages contribuent au relargage de fragments cryptiques qui peuvent être doués d'activité biologique, et peuvent soit favoriser, soit freiner, le 


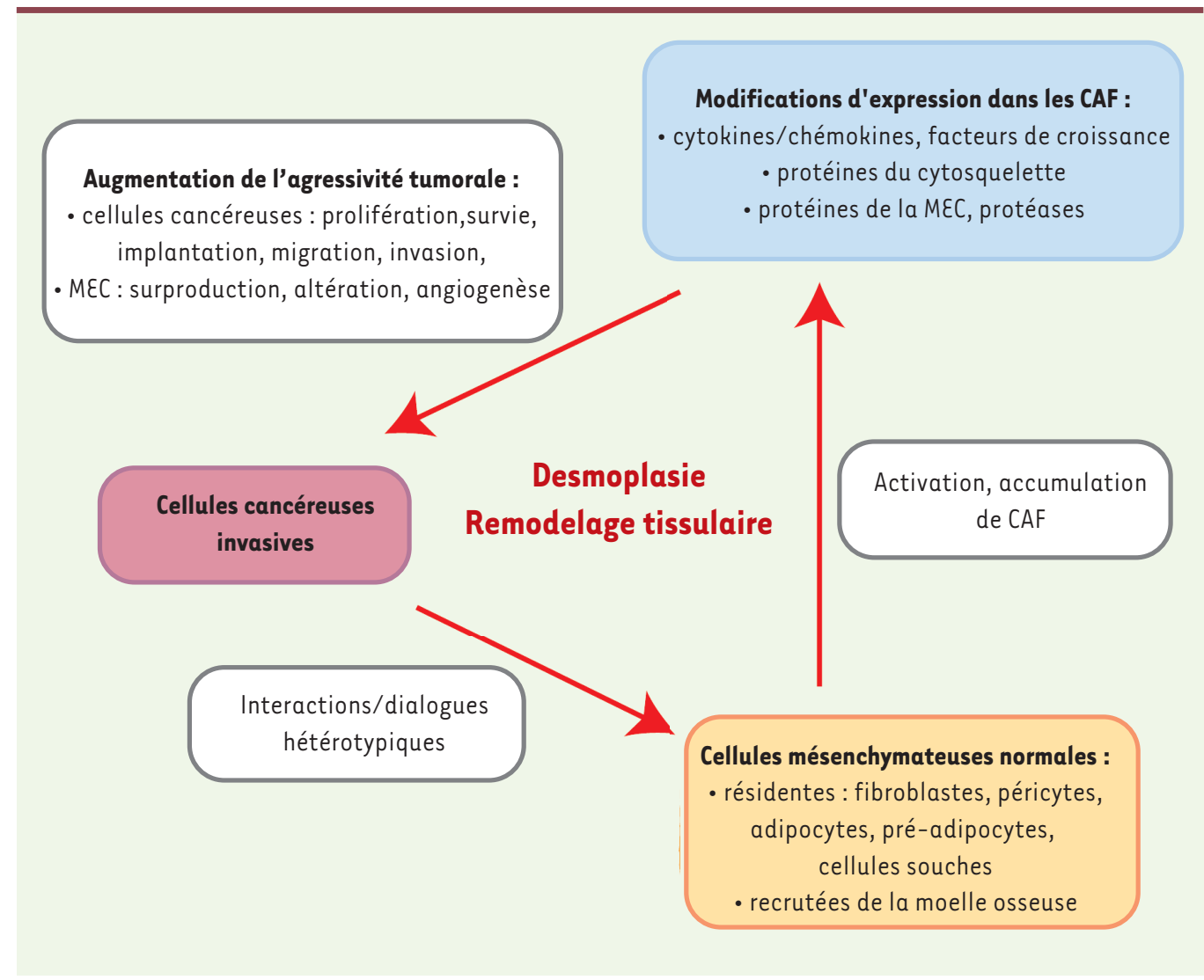

Figure 2. Schéma récapitulant les effets réciproques entre cellules cancéreuses invasives et cellules mésenchymateuses normales. L'interaction/dialogue hétérotypique qui s'établit entre cellules épithéliales cancéreuses et cellules stromales normales est à l'origine des phénomènes de remodelage tissulaire/ desmoplasie. Des cellules stromales normales résidentes, ou recrutées à partir de la moelle osseuse, sont à l'origine de la formation et de l'accumulation de CAF. Ces derniers présentent des phénotypes moléculaires activés caractérisés par des modifications d'expression de nombreux facteurs. Les CAF vont, à leur tour, favoriser la progression tumorale par différents mécanismes biologiques touchant, d’une part, les cellules cancéreuses et, d'autre part, le stroma.

développement tumoral. II existe des récepteurs pour certains de ces fragments à la surface des cellules cancéreuses. Ainsi, la lumcorine, un fragment actif du lumican (un protéoglycane à kératane sulfate), est capable d'inhiber la migration de cellules de mélanomes [19]. À l'inverse, l'endotrophine, un fragment du collagène de type VI, possède des propriétés procancéreuses dans les cancers du sein [20].

La spécificité de substrats des MMP n'est pas limitée aux composants de la MEC. Certaines de ces enzymes peuvent cliver un nombre incalculable de substrats et avoir des fonctions variées. Elles peuvent notamment activer des pro-MMP (pro-MMP2, pro-MMP13, etc.), hydrolyser des protéines à la surface des cellules cancéreuses ( $\varepsilon$-cadhérine, intégrines, etc.), ou encore libérer des facteurs de croissance séquestrés dans la MEC (IGFI [insulin-like growth factor 1]) [21].

\section{Le stroma tumoral : un marqueur pronostique/diagnostique?}

Des études cliniques ont montré que la nature/intensité de la réaction stromale est corrélée à certains facteurs pronostiques classiques. Une forte réaction stromale est associée à une moins bonne survie des patients [22]. Ainsi, des analyses à haut débit ont mis en évidence une signature stromale qui est associée à un mauvais pronostic dans les cancers du sein [23]. L'intérêt d'inclure certaines molécules stromales dans des tests pronostiques est actuellement en cours d'évaluation dans des études cliniques sur les cancers du sein. Par exemple, la MMP1l et la cathepsine L2 (CTSL2) font partie du test Oncotype Dx, une signature génomique de 16 gènes [24].

Enfin, le microenvironnement tumoral peut influencer la réponse des cellules cancéreuses à des thérapies systémiques. Ainsi, une signature stromale prédit la résistance à la chimiothérapie dans

$(\rightarrow)$ Voir la Synthèse de L. Borriello et y.A. DeClerck, page 445 de ce numéro les cancers du sein $[25,37](\rightarrow)$.

\section{Le stroma tumoral : une cible thérapeutique potentielle?}

Comme nous l'avons vu, le stroma doit être considéré non pas comme un tissu de soutien, mais comme faisant partie intégrante des tumeurs solides et directement impliqué dans le développement tumoral. Dans ce contexte, la recherche de thérapies ciblant non plus la cellule cancéreuse, mais le stroma, apparaît donc comme une évidence. L'effet causal de plusieurs 
facteurs stromaux sur le développement des tumeurs ne fait aucun doute, tout du moins à certaines étapes de la maladie. La possibilité d'intervenir sur ces derniers, ou sur des voies de signalisation en amont ou en aval, a donc été envisagée. En effet, les traitements cytotoxiques ciblant l'activité métabolique/proliférative accrue des cellules cancéreuses induisent de nombreux effets secondaires indésirables, voire délétères. Des événements de résistances acquises aux traitements sont fréquents. Afin de remédier à cet état de fait, il a été envisagé de cibler les cellules stromales normales, qui ne sont donc pas accessibles à une plasticité génomique souvent impliquée dans les phénomènes de résistance. De plus, ces thérapies dites cytostatiques auraient des effets secondaires moindres. Ainsi, des inhibiteurs d'angiogenèse et des anti-inflammatoires ont déjà été utilisés avec plus ou moins de succès.

Concernant les CAF et la MEC, plusieurs pistes de développement de thérapies moins agressives ont été explorées. C'est le cas, par exemple, de thérapies ciblant des voies de signalisation paracrines utilisées par les CAF pour influencer les cellules cancéreuses. De plus, les CAF peuvent être directement ciblés, par exemple avec une prodrogue activée par la FAP (fibroblast activation protein), une protéase qu'ils expriment [26]. Parmi les autres cibles potentielles, plusieurs MMP sont intéressantes, et de nombreux inhibiteurs (MMPI) ont été développés au cours de ces 20 dernières années. Cependant, alors que les études précliniques chez l'animal étaient plutôt prometteuses, les essais cliniques se sont révélés décevants pour la plupart [27]. Malgré tout, l'idée de cibler les MMP en thérapie n'est pas abandonnée, et des études sont actuellement menées pour améliorer la spécificité et l'efficience des MMPI, ou encore pour utiliser les MMP grâce à d'autres approches (optimisation de la biodisponibilité des drogues, vaccination, ciblage du domaine hémopexine) [28].

D’une certaine manière, la masse tumorale doit être appréhendée comme une entité, une sorte d'organe aberrant. II convient alors d'imaginer des approches thérapeutiques ciblant ses deux composantes, épithéliale et stromale. Des études précliniques chez l'animal ont montré que l'administration, simultanément à la chimiothérapie, de drogues ciblant le microenvironnement tumoral facilitait la réponse thérapeutique. Certaines associations médicamenteuses sont actuellement en cours d'évaluation. Par exemple, l'administration intraveineuse d'un variant de la hyaluronidase (PEGPH20) est en phase clinique I [29].

\section{Conclusion}

Les recherches récentes en biologie des cancers ont fait émerger un rôle paracrine majeur du stroma dans la progression tumorale, notamment dans les phases d'invasion locale, un prérequis pour la formation des métastases. Ainsi, le concept proposé par Paget dès la fin du $x x^{e}$ siècle de «la graine et du sol » associant à une cellule devenue incontrôlable un terrain propice à sa survie et à son expansion [30-32] reste tout à fait pertinent. Les données issues de l'étude du stroma permettent actuellement d'améliorer les critères pronostiques et diagnostiques dans certains cancers. Cependant, en termes de traitement, bien qu'un certain nombre d'études précliniques aient montré notre capacité à développer des outils thérapeutiques efficaces ciblant des facteurs du stroma, beaucoup reste à faire. Le défi est de disséquer au niveau moléculaire la dynamique réciproque qui s'établit entre les cellules épithéliales cancéreuses et leur microenvironnement, dans le but de faire émerger des cibles stromales potentiellement intéressantes. En effet, malgré les avancées technologiques permettant de mesurer la séquence, le nombre de copies et l'expression de chaque gène dans un tissu donné, la connaissance des mécanismes moléculaires et des voies de signalisation impliqués dans l'interaction/ dialogue entre les cellules cancéreuses et les cellules stromales, notamment via les CAF, n'en est encore qu'à ses balbutiements. Enfin, des approches combinées, ciblant de concert les cellules tumorales et leur microenvironnement, devraient entraîner un bénéfice clinique important ; le but in fine étant, d'une part, d'optimiser et d'individualiser les thérapies et, d'autre part, d'en réduire au maximum les effets secondaires. $\diamond$

\section{SUMMARY}

The tumoral stroma, a breeding ground for cancer cells

Carcinomas are constituted by malignant epithelial cells and the tumor microenvironment also called tumoral stroma. The present non-exhaustive review will focus on cellular and molecular key events implicating cancer-associated fibroblasts (CAF) and the extracellular matrix (ECM) in the stroma remodeling processes occuring during tumor invasive steps. We will also discuss the place of the stroma in the cancerology today, the recent progresses done and its usefulness to design new therapies. $\diamond$

\section{REMERCIEMENTS}

Ce travail a été financé par des fonds provenant de l'Inserm, du CNRS, de l'Association pour la recherche sur le cancer (ARC), de la Ligue nationale française contre le cancer (équipe labellisée Ligue contre le cancer; comités du Haut-Rhin, du Bas-Rhin et de la Haute-Savoie) et de l'Institut national du cancer (INCA; contrat PL-BIO ADIPOK 2011-2013). E. B. était boursière de la Ligue contre le cancer et de l'INCA.

\section{LIENS D'INTÉRÊT}

Les auteurs déclarent n'avoir aucun lien d'intérêt concernant les données publiées dans cet article.

\section{RÉFÉRENCES}

1. De Wever 0, Mareel M. Role of tissue stroma in cancer cell invasion. J Pathol $2003 ; 200: 429-47$. 


\section{RÉFÉRENCES}

2. Mueller MM, Fusenig NE. Friends or foes - bipolar effects of the tumour stroma in cancer. Nat Rev Cancer $2004 ; 4: 839-49$.

3. Lu P, Weaver VM, Werb Z. The extracellular matrix: a dynamic niche in cancer progression. J Cell Biol $2012 ; 196: 395-406$.

4. Bissell MJ, Hines WC. Why don't we get more cancer? A proposed role of the microenvironment in restraining cancer progression. Nat Med $2011 ; 17: 320-9$.

5. Folkman J. Role of angiogenesis in tumor growth and metastasis. Semin Oncol $2002 ; 29: 15-8$.

6. Motrescu ER, Rio MC. Cancer cells, adipocytes and matrix metalloproteinase 11: a vicious tumor progression cycle. Biol Chem 2008 ; 389 : 1037-41.

7. Dirat B, Bochet L, Dabek M, et al. Cancer-associated adipocytes exhibit an activated phenotype and contribute to breast cancer invasion. Cancer Res $2011 ; 71: 2455-65$.

8. Tan J, Buache $\varepsilon$, Chenard MP, et al. Adipocyte is a non-trivial, dynamic partner of breast cance cells. Int J Dev Biol $2011 ; 55: 851-9$.

9. Wang CS, Tetu B. Stromelysin-3 expression by mammary tumor-associated fibroblasts under in vitro breast cancer cell induction. Int J Cancer $2002 ; 99: 792-9$

10. Fukino K, Shen L, Matsumoto S, et al. Combined total genome loss of heterozygosity scan of breast cancer stroma and epithelium reveals multiplicity of stromal targets. Cancer Res 2004 ; $64: 7231-6$.

11. Haviv I, Polyak K, Qiu W, et al. Origin of carcinoma associated fibroblasts. Cell Cycle $2009 ; 8: 589-95$

12. Polanska UM, Orimo A. Carcinoma-associated fibroblasts: non neoplastic tumour-promoting mesenchymal cells. J Cell Physiol 2013 ; 228 : 1651-7.

13. Basset P, Bellocq JP, Lefebvre 0 , et al. Stromelysin-3: a paradigm for stroma-derived factors implicated in carcinoma progression. Crit Rev Oncol Hematol 1997 ; $26: 43-53$.

14. Masson $R$, Lefebvre 0 , Noel $A$, et al. In vivo evidence that the stromelysin-3 metalloproteinase contributes in a paracrine manner to epithelial cell malignancy. J Cell Biol $1998 ; 140: 1535-41$

15. Jacob MP. Matrice extracellulaire et vieillissement vasculaire. Med Sci (Paris) $2006 ; 22: 273-8$.

16. Marastoni $S$, Ligresti $G$, Lorenzon $\varepsilon$, et al. Extracellular matrix: a matter of life and death. Connect Tissue Res 2008 ; 49 : 203-6.

17. Goetz JG, Minguet S, Navarro-Lerida I, et al. Biomechanical remodeling of the microenvironment by stromal caveolin-1 favors tumor invasion and metastasis. Cell $2011 ; 146: 148-63$.

18. Paszek MJ, Zahir N, Johnson KR, et al. Tensional homeostasis and the malignant phenotype. Cancer Cell $2005 ; 8: 241-54$.

19. Brezillon S, Pietraszek K, Maquart FX, et al. Lumican effects in the control of tumour progression and their links with metalloproteinases and integrins. FEBS J 2013;280:2369-81.

20. Park J, Scherer PE. Adipocyte-derived endotrophin promotes malignant tumor progression. J Clin Invest $2012 ; 122: 4243-56$

21. Kessenbrock K, Plaks V, Werb Z. Matrix metalloproteinases: regulators of the tumor microenvironment. Cell $2010 ; 141: 52-67$.

22. Finak $\mathrm{G}$, Bertos $\mathrm{N}$, Pepin $\mathrm{F}$, et al. Stromal gene expression predicts clinical outcome in breast cancer. Nat Med $2008 ; 14: 518-27$.
23. Beck AH, Espinosa I, Gilks CB, et al. The fibromatosis signature defines a robust stromal response in breast carcinoma. Lab Invest 2008 ; 88 : 591-601.

24. Paik S, Shak S, Tang $G$, et al. A multigene assay to predict recurrence of tamoxifentreated, node-negative breast cancer. N EnglJ Med 2004 ; 351 : 2817-26.

25. Farmer $\mathrm{P}$, Bonnefoi $\mathrm{H}$, Anderle $\mathrm{P}$, et al. A stroma-related gene signature predicts resistance to neoadjuvant chemotherapy in breast cancer. Nat Med $2009 ; 15: 68-74$

26. Brennen WN, Rosen DM, Wang $\mathrm{H}$, et al. Targeting carcinoma-associated fibroblasts within the tumor stroma with a fibroblast activation proteinactivated prodrug. J Natl Cancer Inst 2012; 104 : 1320-34

27. Coussens LM, Fingleton B, Matrisian LM. Matrix metalloproteinase inhibitors and cancer: trials and tribulations. Science $2002 ; 295: 2387-92$.

28. Buache $\varepsilon$, Rio MC. New prospects for matrix metalloproteinase targeting in cancer Therapy. In : Behrendt M, ed. Matrix proteases in health and disease. New York : Wiley-VCH, 2012 : 373-88.

29. Thompson CB, Shepard HM, O'Connor PM, et al. Enzymatic depletion of tumor hyaluronan induces antitumor responses in preclinical animal models. Mol Cancer Ther $2010 ; 9: 3052-64$.

30. Paget $\mathrm{S}$. The distribution of secondary growths in cancer of the breast. Lancet $1889 ; 133: 571-3$.

31. Fridman WH, Sautès-Fridman C. Le microenvironnement tumoral : matrice nourricière, champ de bataille etcible thérapeutique des cancers. Med Sci (Paris) $2014 ; 30: 359-65$.

32. Provot $\mathrm{S}$. Contrôle de la croissance et de la dissémination tumorales par le microenvironnement : certitudes et hypothèses émergentes. Med Sci (Paris) $2014 ; 30: 366-71$

33. Albrengues J, Meneguzzi G, Gaggioli C. L'invasion des cellules tumorales : quand les fibroblastes s'en mêlent. Med Sci (Paris) 2014 ; 30 : 391-7.

34. Le Guellec $S$, Duprez-Paumier R, Lacroix-Triki M. Microenvironnement tumoral : la vision du pathologiste. Med Sci (Paris) 2014 ; $30: 372-7$.

35. Azzi S, Gavard J. Vaisseaux sanguins et tumeurs ou l'art du dialogue. Med Sci (Paris) $2014 ; 30: 408-14$.

36. Garrido-Urbani S, Jaquet V, Imhof BA. ERO, NADPH oxydases et vascularisation des tumeurs. Med Sci (Paris) $2014 ; 30: 415-21$

37. Laurent V, Nieto L, Philippe Valet P, Muller C. Tissu adipeux et cancer : une association à haut risque. Med Sci (Paris) $2014 ; 30: 398-404$.

38. Borriello L, DeClerck YA. Le microenvironnement tumoral et la résistance thérapeutique. Med Sci (Paris) $2014 ; 30: 445-51$.

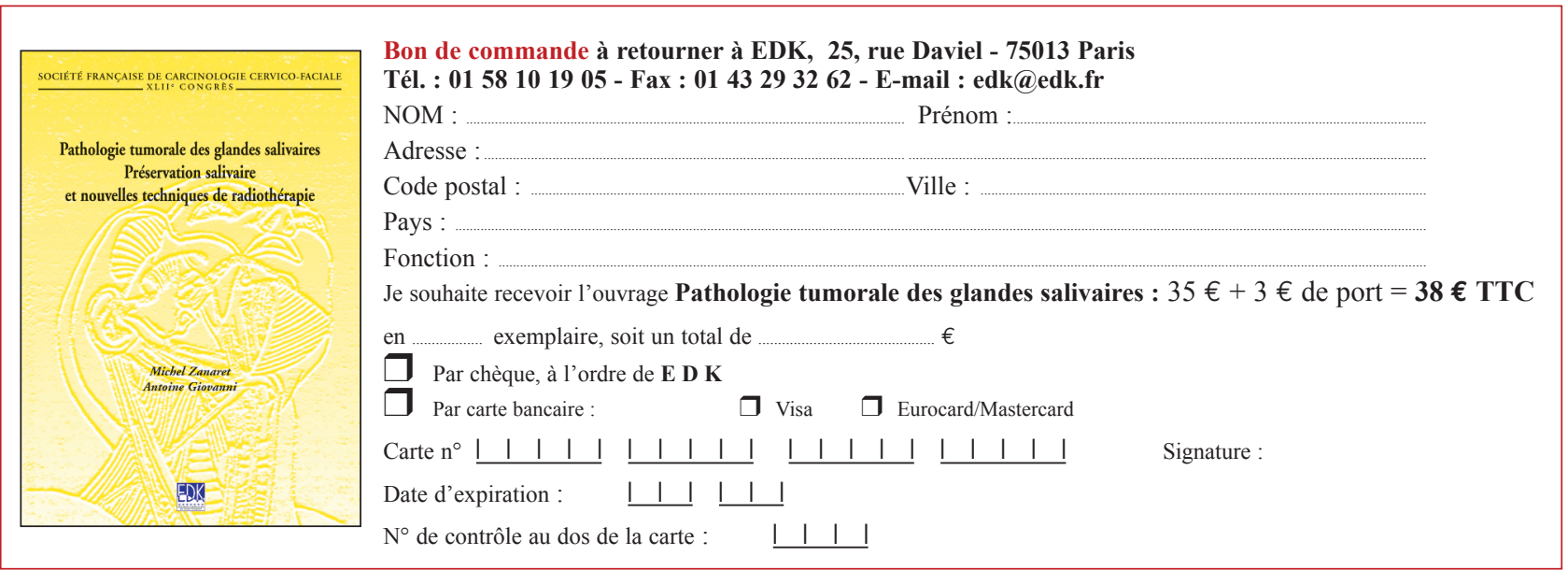

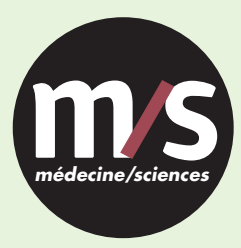

Tarifs d'abonnement $\mathrm{m} / \mathrm{s}-2014$

Abonnez-vous

à médecine/sciences
$>$ Grâce à $m / s$, vivez en direct les progrès des sciences biologiques et médicales



\section{TIRÉS À PART}

M.C. Rio 